\title{
Smart Sensing Technologies and Their Applications in Civil Infrastructures
}

\author{
Hong-Hu Zhu, ${ }^{1}$ Fei Dai, ${ }^{2}$ Zhenhua Zhu, ${ }^{3}$ Tuan Guo, ${ }^{4}$ and Xiao-Wei Ye ${ }^{5}$ \\ ${ }^{1}$ School of Earth Sciences and Engineering, Nanjing University, Nanjing 210023, China \\ ${ }^{2}$ Department of Civil and Environmental Engineering, West Virginia University, Morgantown, WV 26506, USA \\ ${ }^{3}$ Department of Building, Civil and Environmental Engineering, Concordia University, Montreal, QC, Canada H3G $1 M 8$ \\ ${ }^{4}$ Institute of Photonics Technology, Jinan University, Guangzhou 510632, China \\ ${ }^{5}$ Department of Civil Engineering, Zhejiang University, Hangzhou 310058, China
}

Correspondence should be addressed to Hong-Hu Zhu; zhh@nju.edu.cn

Received 27 April 2015; Accepted 27 April 2015

Copyright (c) 2015 Hong-Hu Zhu et al. This is an open access article distributed under the Creative Commons Attribution License, which permits unrestricted use, distribution, and reproduction in any medium, provided the original work is properly cited.

With the rapid development of urbanization in some developing countries such as China, India, and Brazil, numerous huge civil infrastructures have been built in recent years. The performance of these structures during construction, operation, maintenance, and repair becomes a major concern. In order to accurately assess the health condition of engineering structures and prevent potential failure, a variety of smart sensing technologies and methods have been successfully developed in the past few decades, such as fiber optic sensing (FOS), wireless sensor network (WSN), acoustic emission (AE), time domain reflectometry (TDR), microelectromechanical system (MEMS), global position system (GPS), particle image velocimetry (PIV), and laser scanning. Due to their inherent advantages, these technologies have played an increasingly important role in health monitoring of different types of civil infrastructures.

The aim of this special issue is to present original research articles on smart sensing technologies and their applications in civil infrastructures. The topics cover various aspects including novel smart sensing mechanisms and devices for civil infrastructures; applications of smart sensors and sensing systems in civil infrastructures; in-field implementation of structural health monitoring and diagnosis systems; supporting technologies and methodologies in monitoring civil infrastructures, such as real-time data acquisition and processing, data transferring, remote management, and performance evaluation; and typical case study of sensing applications and civil infrastructures monitoring.
The papers in this special issue are expected to provide indepth insights into the development and applications of smart sensing technologies in civil infrastructures and be useful for civil engineering practitioners and researchers.

\section{Acknowledgments}

The guest editors of this special issue would like to express their sincere gratitude to all the authors and anonymous reviewers who have generously contributed to this special issue.

Hong-Hu Zhu

Fei Dai

Zhenhua Zhu

Tuan Guo

Xiao-Wei Ye 

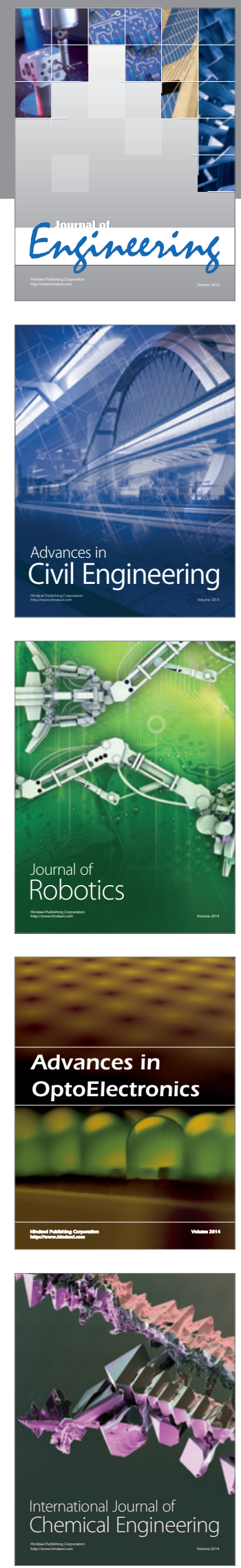

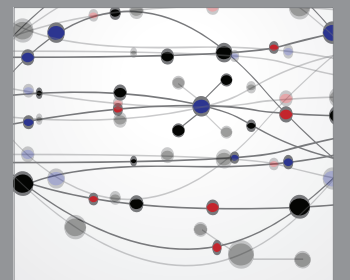

The Scientific World Journal
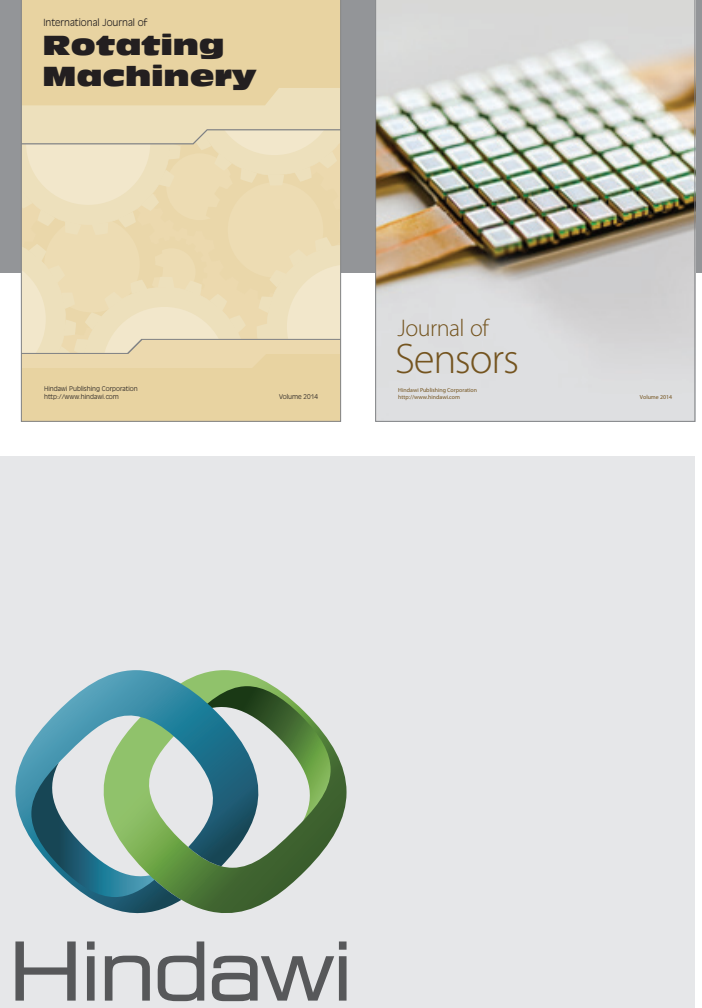

Submit your manuscripts at http://www.hindawi.com
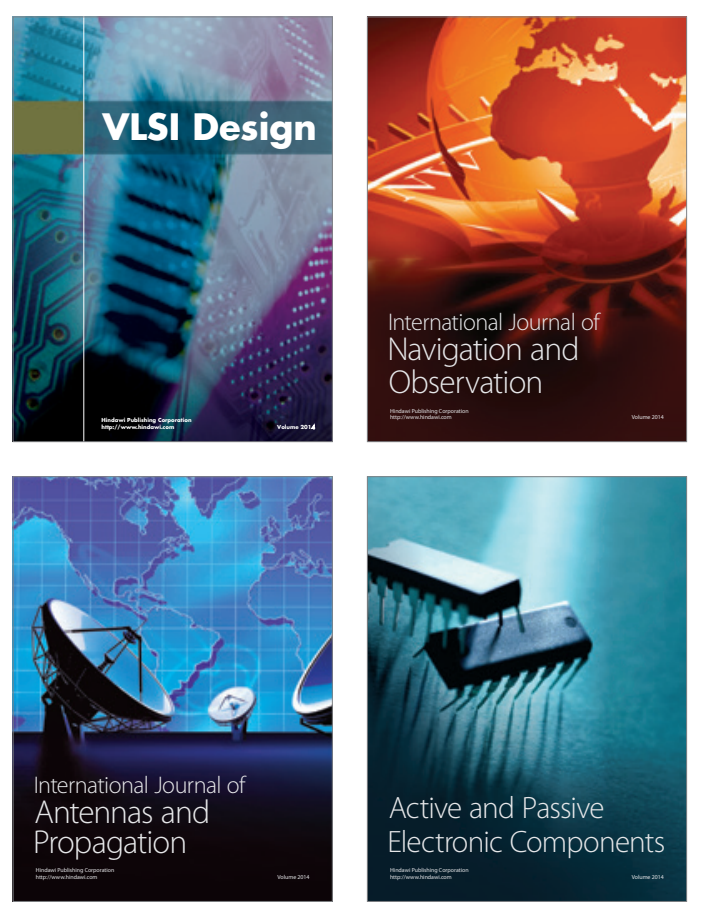
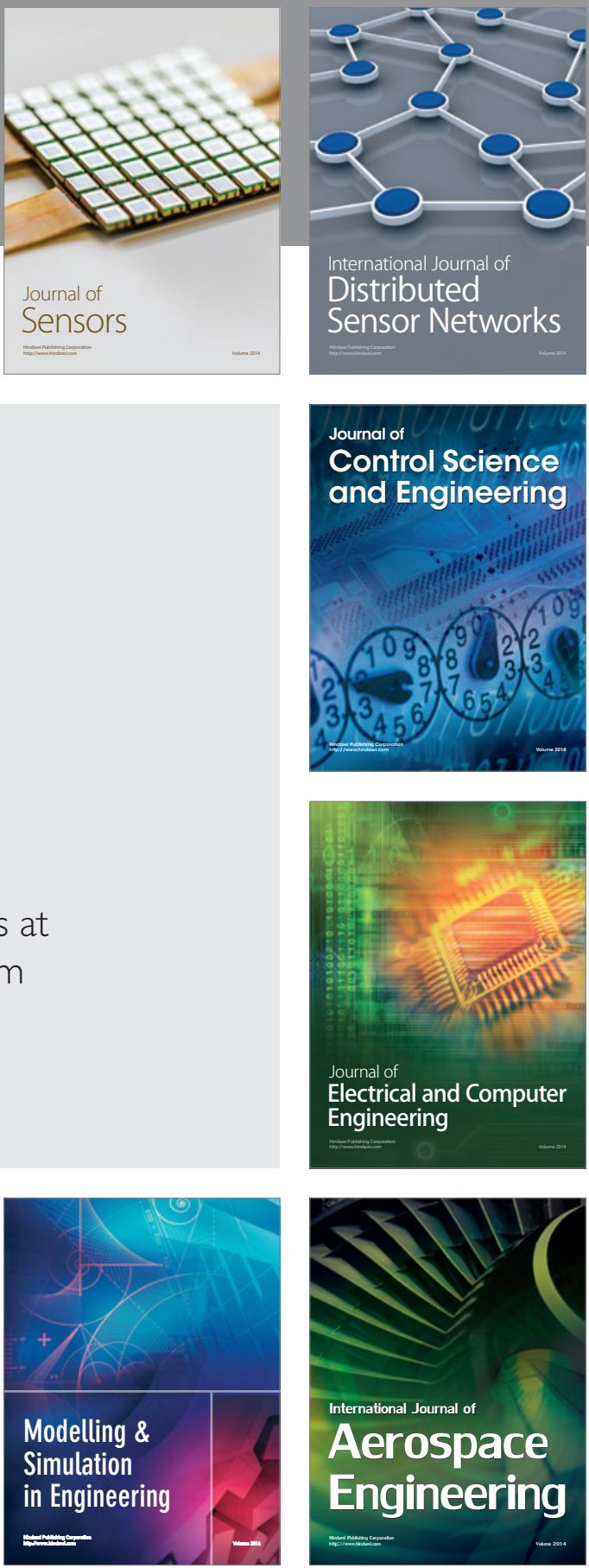

Journal of

Control Science

and Engineering
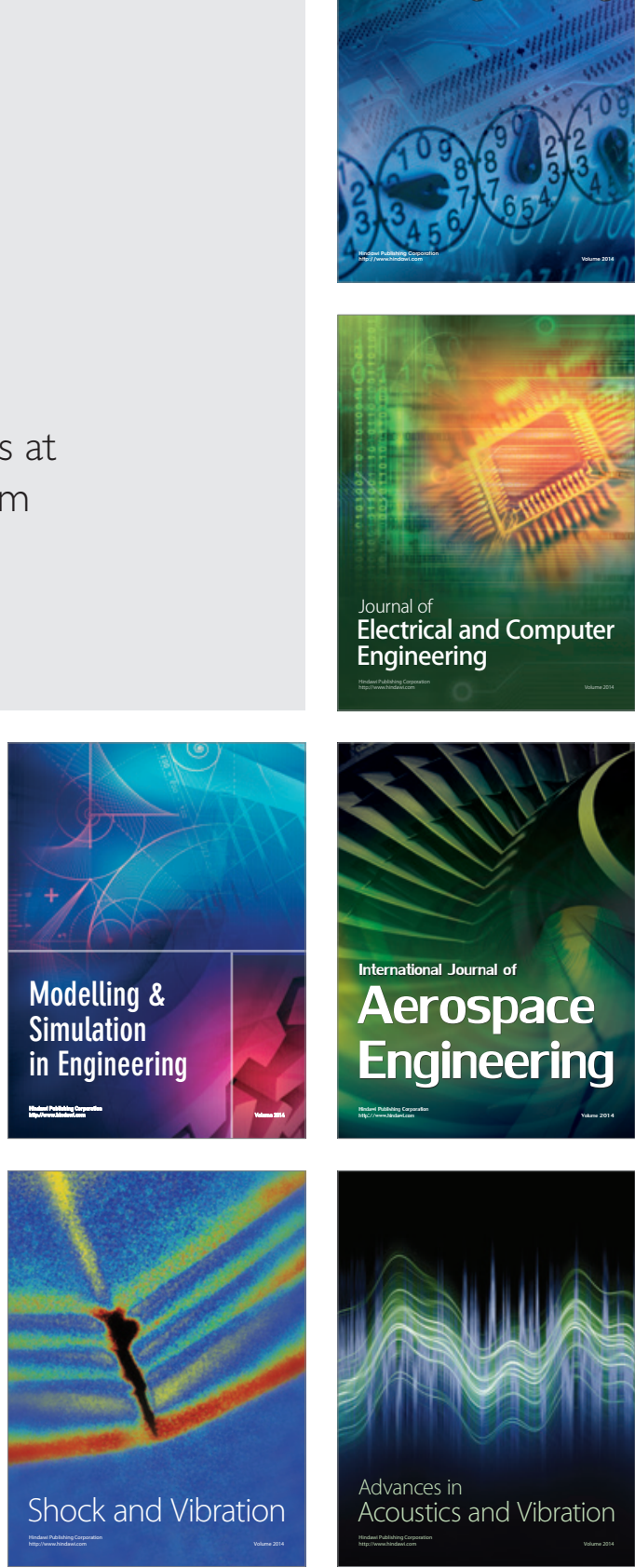